\title{
Stress fracture of the thoracic spine in a male rugby player: a case report
}

\author{
A Shafik, ${ }^{1,2}$ BSc (Hons) Sports and Exercise Sci; K Schwabe, ${ }^{1,2}$ MBChB, MPhil (Sport and Exercise Med); R de Villiers, ${ }^{\mathbf{4}}$ MBChB \\ MMed (Rad. D); J T Viljoen, 1, 2, 3 BSc (Hons) Physio, MPhil (Exercise Sci); W Derman, 1, 2 MBChB, BSc (Med) (Hon), PhD, FFIMS. \\ ${ }^{1}$ Institute of Sport and Exercise Medicine, Division of Orthopedics, Faculty of Medicine and Health Sciences, Stellenbosch University, South Africa \\ ${ }^{2}$ IOC Research Centre, Cape Town, South Africa \\ ${ }^{3}$ Campus Health Service, Stellenbosch University, South Africa \\ ${ }^{4}$ Winelands Radiology, Institute of Orthopaedics and Rheumatology, Stellenbosch, South Africa
}

Corresponding author: J T Viljoen (jtviljoen@sun.ac.za)

This case report discusses a stress fracture of the thoracic spine in a professional South African rugby union player. This is a rare anatomical location for this type of injury in this population and has not previously been described. Physicians should be aware that performance of rugby specific movements may lead to rare stress fractures in certain anatomic locations.

Keywords: Overuse injury, sport, bone, back

S Afr J Sports Med 2018; 30:1-2. DOI: 10.17159/2078-516X/2018/v30i1a4592

Professional rugby union players are at high risk of spinal injuries due to scrummaging, tackling and weight training activities.[1] Spondylolysis or stress fractures of the vertebral pars interarticularis or other spinal stress fractures are uncommon injuries in rugby which, to the best of these authors' knowledge, have only previously been described in the lumbar spine and sacrum. ${ }^{[2,3]}$ Here the case of a stress fracture in the thoracic spine of a professional rugby union player is presented.

\section{Case report}

\section{History}

A 21-year-old male professional tighthead prop presented with a three-month history of nocturnal right-sided midthoracic dorsal pain, which started progressively increasing in severity and was further aggravated by playing rugby. The only injury the player could recall was a sternal contusion when he landed on a ball three months prior to the initiation of the back pain. Furthermore, the patient's medical history was unremarkable, with no additional symptoms.

\section{Examination}

The initial clinical examination demonstrated mild scoliosis and midline lower thoracic spinal tenderness to palpation. Provocative manoeuvres recreating right lower thoracic pain included standing, lateral rib cage compression, forward flexion (fingertips reached mid-tibial level), bilateral rotation and lateral flexion. Moreover, resisted flexion of the right hip caused lower thoracic pain. Additional examinations revealed nothing further of significance. At this point, a differential diagnosis of soft tissue injury, bony fracture (rib or vertebra), arthritis, infective process and malignancy were considered.

\section{Investigations: Blood tests and imaging studies}

The patient was initially referred for standard plain radiographs (X-rays) of the thoracic spine. These showed mild dorso-lumbar scoliosis without any further abnormalities. Additionally, a comprehensive laboratory blood workup was requested consisting out of a full blood count, an assessment of urea and electrolytes, CRP (C-reactive protein) levels, ESR (erythrocyte sedimentation rate) as well as serum protein electrophoresis and serum 25-hydroxy vitamin D concentration. All blood results proved to be normal with the exception of slightly elevated phosphate concentration and a low serum 25 -hydroxy vitamin D concentration at $22 \mathrm{ng} / \mathrm{mL}$.

Further investigation using magnetic resonance imaging (MRI) revealed bone oedema of the T6, T7 and $\mathrm{T} 8$ pedicles and superior articular processes with early degenerative disc disease at the cervico-thoracic junction (Fig. 1A and 1B). Computed tomography (CT) confirmed chronic bone stress reactions on the right $\mathrm{T} 7, \mathrm{~T} 8, \mathrm{~T} 9, \mathrm{~T} 10$ pedicles and superior articular processes. Chronic bone stress fractures of the right T8 and T9 superior articular processes were also found (Fig. 1C). A technetium bone scan plus single-photon emission computed tomography (SPECT) showed increased uptake in the medial aspect of the T8, T9 and, to a lesser extent, of the T10 pedicles on the right. The uptake extended onto the superior articular processes of the respective vertebrae on the right. Based on these findings, the diagnosis of bone stress injury with stress fractures of the right $\mathrm{T} 8$ and $\mathrm{T} 9$ pedicles was made.

\section{Treatment}

The athlete's most important aspect of his treatment was physical rest from all aggravating exercise, with carefully scheduled reassessment of repeated clinical examinations. Once asymptomatic, the athlete participated in a gradual incremental return to individual training and fitness utilising deep water aqua therapy and swimming in warm water before rejoining the squad for further comprehensive sport specific rehabilitation, and eventual return to play. Oral vitamin D supplementation in the form of Calciferol (50 000 IU weekly) was administered. His blood concentrations had returned to normal when remeasured after two months. 

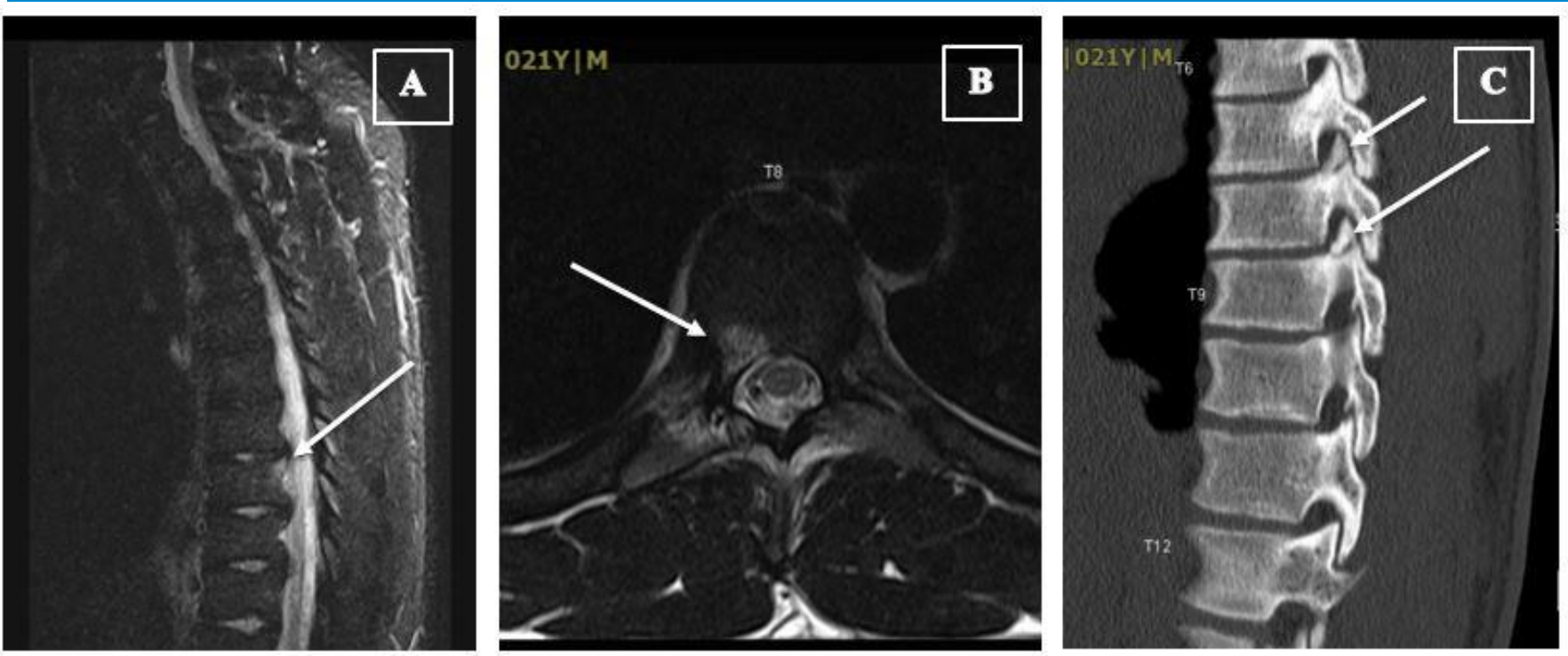

Fig. 1A. Magnetic resonance imaging of thoracic spine (sagittal view), showing bone marrow oedema of the pedicle.

Fig. 1B. Magnetic resonance imaging of T8 vertebrae (axial view) revealing bone marrow oedema in relation to the stress fracture.

Fig. 1C. Computed tomography of thoracic spine (sagittal view) showing significant, unilateral sclerosis of the pedicle and superior articular process with associated fracture lines.

\section{Discussion}

A rare case of a stress fracture of the thoracic vertebrae in a professional rugby union player was presented in this case study. Spondylolysis is thought to occur due to repeated stress on the vertebra and most commonly occurs in the lumbar spine in rugby players. ${ }^{[2]}$ To date, the only thoracic spinal fracture reported in a rugby player was a traumatic fracture that occurred in a rugby league game. ${ }^{[4]}$ According to these authors, this is the first case study describing a thoracic stress fracture in a rugby union game. This injury should be considered as a differential diagnosis when evaluating rugby players, especially in front row players, who are more susceptible to spinal injuries due to the repeated extreme force experienced during scrum engagement. ${ }^{[5]}$

The pathogenesis of the case is still unclear. Consultant opinion from a spinal orthopaedic specialist was discussed with the athlete and it was suggested that the injury might have arisen due to weakness of the athlete's left shoulder girdle, which resulted in difficulty with binding. The athlete confirmed that his thoracic trunk did indeed flex to the right when scrumming which could explain the unusual right-sided bony stress response. It was suggested that the muscle strength of both shoulders be evaluated and a series of ongoing strengthening exercises be considered if deficits were identified. Further suggestions for management included playing in the loosehead position and use of his stronger right arm for firm binding.

Due to the rarity of this condition, it could be missed and therefore team physicians need to be aware of the possibility of this type of injury in the case of dorsal pain. This is even more crucial in young rugby union players where failure to detect this early on in a diagnosis may result in acute-onchronic injuries throughout a player's career. Finally, it is of interest to note that a series of lumbar spine stress fractures caused the French National Professional Rugby League to include X-ray and MRI of the cervical and lumbar spine as part of systematic screening in academy centres to detect congenital or developmental anomalies that may potentially increase spinal injuries for aspiring professionals. ${ }^{[2]}$

\section{Conclusion}

A thoracic spinal injury, especially bone stress, within sports medicine is a rare but important pathology to consider as a differential diagnosis. A clinician assessing and managing rugby union players should consider this rare injury when confronted with ongoing thoracic pain, particularly in scrumming players. MRI and CT form an important modality for diagnosis when a suspicion of injury for these conditions is raised.

Study funding and conflict of interest: The authors report no funding or conflict of interest.

\section{References}

1. Fuller CW, Brooks JH, Kemp SP. Spinal injuries in professional rugby union: a prospective cohort study. Clin J Sport Med 2007; 17(1):10-16. [doi: 10.1097/JSM.0b013e31802e9c28]

2. Castinel BH, Adam P, Prat C. A stress fracture of the lumbar spine in a professional rugby player. Br J Sports Med 2007; 41(5): 337-338. [doi: 10.1136/bjsm.2006.032789]

3. Takahashi Y, Kobayshi T, Miyakoshi N, et al. Sacral stress fracture in an amateur rugby player: a case report. J Med Case Rep 2016; 10(1):327. [doi: 10.1186/s13256- 016- 1120-3]

4. Geffen S, Gibbs N, Geffen L. Thoracic spinal fracture in a rugby league footballer. Clin J Sport Med 1997; 7(2): 144-146. [PMID: 9113434]

5. Trewartha G, Preatoni E, England, ME, et al. Injury and biomechanical perspectives on the rugby scrum: a review of the literature. Br J Sports Med 2015; 49(7): 425-433. [doi: 10.1136/bjsports-2013-092972] 